\title{
Nonpsychotic Psychiatric Disorders in Juvenile Myoclonic Epilepsy
}

\author{
Polina V. Moskaleva; Olga S. Shilkina, MD; Ivan P. Artyukhov, PhD, ScD; Irina G. \\ Strotskaya, MD; Diana V. Dmitrenko, PhD, ScD; Natalia A. Shnayder, PhD, ScD* \\ V.F. Voyno-Yasenetsky Krasnoyarsk State Medical University \\ Krasnoyarsk, The Russian Federation
}

\begin{abstract}
The association of epilepsy with mental illness has been described in recent years. Scientists are trying to relate certain epilepsies, such as juvenile myoclonic epilepsy (JME), with certain personality traits marked by emotional instability. The goal of this review is to evaluate the scientific literature about nonpsychotic psychiatric disorders in JME patients, the most common form of idiopathic generalized epilepsy (IGE). Data in this review were collected through an extensive literature search of available full-text publications in PubMed, Springer, Clinical Keys and eLIBRARY.RU databases. Comorbid personality and nonpsychotic psychiatric disorders are a common and interdisciplinary problem in JME management. The disorders in patients with JME often go undiagnosed and hence untreated. Therefore, this problem requires further and extensive investigation. (International Journal of Biomedicine. 2017;7(2):85-90.)
\end{abstract}

Key Words: juvenile myoclonic epilepsy • personality disorders • nonpsychotic psychiatric disorders • antiepileptic drugs

\section{Abbreviations}

ADCME, autosomal dominant cortical tremor, myoclonus, and epilepsy; AEDs, antiepileptic drugs; BIS, Barratt impulsiveness scale; DSM, Diagnostic and Statistical Manual of Mental Disorders; IGE, idiopathic generalized epilepsy; JME, juvenile myoclonic epilepsy; PDs, personality disorders; SAS, social adjustment scale; TLE, temporal lobe epilepsy.

\section{Introduction}

JME is one of the most widespread forms of IGE in adolescents and young adults. JME is characterized by the classical Herpin-Janz triad: frequent myoclonic jerks, most often of the upper limbs; possible generalized tonic-clonic seizures; and, less commonly, absences. ${ }^{(1)}$ Seizures are often preceded by stress and a disturbance in the circadian rhythm of sleep with a predominance of seizures after awakening ( $83 \%$ of patients); a frequent cause of these seizures is sleep deprivation (77\%). ${ }^{(2)}$ A decrease in the frequency and severity of seizures depends not only on the doctor, who needs to choose the therapy correctly, but also on the patient, who in

*Corresponding author: Prof. Natalia A. Shnayder, PhD, ScD; Head of the Neurological Center of Epileptology, Neurogenetics and Brain Research of the University Clinic of V.F. Voino-Yasenetsky Krasnoyarsk State Medical University. Krasnoyarsk, The Russian Federation. E-mail: nataliashnayder@gmail.com turn adheres to the sleep-wakefulness regime, who prevents menstrual irregularities, and who avoids strong emotional shakes, and other behaviors that might cause stress.

Despite recognizing the above factors, which cause more frequent seizures and worsening of the general condition, many patients cannot cope with their emotions singly; they note a decrease in stress resistance, and therefore complain about the difficulties of falling asleep, resulting in an undesirable lack of sleep.

Janz and Christian, who first distinguished JME as a syndrome in $1957,{ }^{(3)}$ in their classic characterization of JME described the distinctive personality features of such patients as unsteadiness, lack of discipline, hedonism, and an indifference to their disease. ${ }^{(4,5)}$ The authors also identified lack of drive and endurance, unstable self-concept, and rapidly changing affect and mood states, that, according to them, often complicated treatment of JME. ${ }^{(4,6)}$ People with personality disorders (PDs) often experience other mental health problems, especially depression and substance misuse. 
Executive functions refer to processes involved in the elaboration of cognitive and behavioral responses and strategies for the attainment of immediate or future goals. ${ }^{(7)}$ JME patients often demonstrate non-compliance with the doctor's prescriptions. Even if they say that they are doing everything, in fact they forget to attend control visits and to take their medication regularly. ${ }^{(8)}$ Such inadequate behavior (aggravation of executive and intellectual functions) may be associated with long-term anxiety. Thus, the harmful effects on cognition of an early onset of seizure and longer duration of the disease have been well demonstrated. ${ }^{(9)}$

The cause of such disorders may be concomitant nonpsychotic psychiatric disorders, often described with JME. These include various types of anxiety and mood disorders: generalized anxiety disorder, phobias, depression, dysthymia, and psychosomatic disorders. ${ }^{(10)}$ In addition, the presence of seizures in social circumstances reduces social interaction and compromises the quality of interpersonal relationships. ${ }^{(11)}$ The prevalence of psychiatric disorders in nearly one-third of JME patients is similar to that reported in patients with temporal lobe epilepsy, where psychiatric aspects have been more commonly studied. ${ }^{(12)}$ Such disorders further aggravate the patient's condition, closing the vicious circle.

The association of epilepsy with mental illness has been recently described. Scientists are trying to relate certain epilepsies, such as JME, with certain personality traits marked by emotional instability. ${ }^{(13)}$ Furthermore, comorbid personality and nonpsychotic psychiatric disorders in JME, which have been increasingly appearing in the literature recently, are also associated with higher seizure frequency and less seizure control. $^{(14)}$

The goal of this review is to evaluate the scientific literature about nonpsychotic psychiatric disorders in JME patients.

\section{Methods}

Data in this review were collected through an extensive literature search of available full-text publications in PubMed, Springer, Clinical Keys and eLIBRARY.RU databases. We considered studies published from 2010 to 2017 and several earlier works referred to us by other authors. "Juvenile myoclonic epilepsy," "psychiatric disorders," "anxiety," and "impulsivity" were used as keywords. In general, for a specified period we identified 34 publications dealing with the problem of anxiety and depression with JME in the foreign databases. Only 16 of these publications have been included in the review based on search criteria.

\section{Results}

PDs become distinct during adolescence or earlier and continue throughout life. The peak of the JME onset comes at the age of 14 to 16 years, with a range of 8-26 years. ${ }^{(2)}$ Thus, the time periods of the beginning and development of these manifestations are the same, and we can assume that their occurrence is interrelated. Patients with JME inevitably face difficulties in life and employment, such as family problems, reduced social interactions, decreased job opportunities, and low self-esteem. Therefore, doctors often find them anxious and depressed, even with controlled seizures. ${ }^{(11)}$

Studies available in the literature can be divided into two different approaches to PDs in JME: clinical observation, and categorical classification according to the DSM. The first method, clinical observation, is subjective, depends on the doctor's personal perception of the patient and reveals the characteristic personality traits. The second method is not directed at personality traits; rather, it categorizes PDs, distinguishing 10 different subtypes of PDs as well as depressive $\mathrm{PD}$ and passive aggressive $\mathrm{PD}$, which are grouped into three clusters based on their descriptive similarities. ${ }^{(6,15)}$

\section{Personality traits in JME}

Impulsivity is a multidimensional and complex personality trait that influences the pathogenesis of the course and clinical severity of several disorders. A pathological increase in impulsivity is the reason for impulse control disorders and hence impulsive PDs. ${ }^{(16)}$

Patients with JME, compared to people without the disorder, have a higher expression of impulsive personality traits that demand early recognition to avoid further consequences and facilitate social insertion, consequently avoiding future stigma. ${ }^{(6)}$

S. Moschetta et al. made the first study addressing the social functioning of 42 patients with JME using SAS (a standardized instrument using objective questions about the subject's life) and demonstrated that JME patients had worse performance than controls, considering overall social adjustment, work, and family relationships. They demonstrated that higher seizure frequency and impulsive traits, but not cognitive performance, were correlated with worse social adjustment, unlike TLE, in which cognitive deficits are very relevant (attention and verbal memory dysfunction were correlated with social adjustment), therefore recognizing the impact of higher seizure frequency on social adjustment. ${ }^{(11)}$

P. Rzezak et al., taking into consideration the necessity of different neuropsychological approaches to distinct epileptic syndromes, studied impulsivity with the help of BIS-11. They compared 20 patients with JME, 20 with TLE, and 26 healthy controls and showed higher BIS scores in all domains of impulsivity (i.e., motor, attentional, and lack of planning) in JME patients. ${ }^{(17)}$

Impulsivity is a temperament feature that results from a combination of low harm avoidance and high novelty seeking, hence a high risk-seeking predisposition in subjects with a low level of temperamental inhibition leads to an impulsive temperament (which is a highly heritable personality trait). $\left.{ }^{(6,18}\right)$

E. Congton et al., in recent study, highlighted the heritability of impulsivity-familial transmission of impulsivity - within and outside the DSM-IV categories of mental disorders, which emphasizes the idea that the impulsivity trait may not be diagnosable by standard psychiatric investigation, as it may not represent a PD. ${ }^{(6,19)}$

The deficit of executive functions and decision-making behavior is also not always determined in conventional behavioral measures, but neuropsychological deficits were 
detected in the imaging studies and more challenging frontal lobe tasks. Br. Wandschneider et al., explaining the risktaking impulsive behavior of 21 patients with JME, suggested frontal lobe dysfunction. This was corroborated by reports on impaired working memory and executive functions, functional and microstructural changes within the medial and dorsolateral prefrontal cortex. ${ }^{(1)}$ They characterized decisionmaking behavior in JME and its neuronal correlates using functional magnetic resonance imaging and concluded that there is a greater proportion of patients with seizures than seizure-free patients having difficulties making advantageous decisions, but no difference in performance between seizurefree patients and controls. Such results add further evidence that continuous seizure activity affects daily life decision making (sign of impulsivity). ${ }^{(20)}$

Poor impulse control results in inadequate behaviors and predicts greater difficulties in solving social problems and acquiring social skills. It has been shown that better impulse control is significantly associated with higher social functioning in JME patients..$^{(11)}$

\section{Nonpsychotic psychiatric disorders in JME}

The 10 official PD subtypes, which are identified in the DSM-IV, are grouped into 3 clusters based on their descriptive similarities ${ }^{(6)}$ The patients with JME most often exhibit cluster B disorders - histrionic, narcissistic, antisocial, borderlineand are characterized by emotional instability, immaturity, lack of discipline, and rapid mood changes. Patients with these characteristics have been associated with a worse seizure outcome. ${ }^{(21)}$

The problem of the categorical method is that many patients who meet criteria for one PD may also meet criteria for other PDs in different clusters. Also, the method does not take into account the manifestations of impulsivity - the most frequent personality traits in JME ${ }^{(19)}$ However, the presence of personality traits may indicate a risk of developing personality disorders, since a combination of traits often leads to the manifestation of such disorders.

The patients with PDs cluster B often demonstrated the executive dysfunctions. J. Walsh et al., trying to find out why JME patients did not observe doctor's prescriptions, demonstrated that abnormal personality traits and psychiatric disorders are associated with worse intellectual and executive functioning, because half of the cohort of their patients exhibited moderate to severe anxiety symptoms. People with JME and higher neuroticism scores reported more anxiety symptoms and more concentration and motor difficulties compared to those with lower scores. Therefore, they concluded that specific patterns of executive dysfunction are related to maladaptive behavior in JME, the cause of which is anxiety. ${ }^{(5)}$

A. Coppola et al. studied psychiatric comorbidities in patients with ADCME and JME. They analyzed both state anxiety (reflecting various subjective and somatic manifestations of anxiety at a given moment) and trait anxiety (stable individual differences in anxiety proneness, a personality trait). State anxiety was more prevalent in ADCME patients compared to JME patients and healthy controls.
However, the anxiety trait was prevalent in both patients with ADCME and patients with JME, compared to healthy controls, suggesting that it might be a common trait of patients with epilepsy. The myoclonus score positively correlated with both state and trait anxiety, which can be explained by the chronic excitation of the cerebello-thalamo-cortical circuits involving the basal ganglia. In addition, the correlation with the duration of the disease and not with the number of seizures confirmed the assumption. Therefore, the authors conclude that anxiety represents a consequence of cortical hyperexcitability. ${ }^{(22)}$

Devrimsel Harika Ertem et al. also compared JME with other types of epilepsy. They found that $37 \%$ of JME patients had comorbid psychiatric disorders, such as anxiety disorders $(17 \%)$, mood disorders (13\%) and psychotic disorders (7\%). However, their results showed that there were no statistical differences in terms of type of the psychiatric disorders between JME and TLE, which suggested that psychiatric disorders might not be specific to the type of epileptic syndromes; however, such disorders often occurred and affected the quality of life, especially significantly attention and concentration problems in JME patients with a mood disorder. ${ }^{(23)}$

Sh. Somayajula et al. studied the association between clinical factors and the two major PDs (anxiety disorders and depressive disorders) in $165 \mathrm{JME}$ patients and the implications for their psychosocial and socioprofessional integration. The 77(46.6\%) patients were diagnosed with PDs; while 50 were categorized as having anxiety disorders, 27 patients had depressive disorders: depression is more common in the elderly, and anxiety is more common in young people. They concluded that lower emotional well-being was associated with anxiety disorders. It was shown that JME patients with depressive disorders have lower social functioning scores, especially patients with marital dissatisfaction and negative marital quality. ${ }^{(14)}$

Increased doses of AEDs, rapid initiation, and polytherapy entail an increased risk. In general, the negative effects of AEDs are less than the sum total of other factors and are usually reversible. ${ }^{(24)} \mathrm{R}$. Thomas et al. examined 60 patients with drug-refractory JME and concluded that the patients were profoundly impaired across the range of tests evaluating intellectual function, language and naming, executive function, the impact of epilepsy, and AED side effects. Eighty-three percent of patients exhibited frank executive dysfunction, which was moderate to severe in $66 \%$. A high prevalence of neurotoxicity symptoms, such as fatigue and poorer functioning across intellectual and memory tests, was also identified. ${ }^{(25)}$

\section{Discussion}

Persons with epilepsy and associated brain lesions have greater psychiatric comorbidity as compared to those without brain lesions. ${ }^{(26)}$ However, JME is commonly associated with psychiatric manifestations, like depression, anxiety, attention deficit hyperkinetic disorder, conduct disorder and aggressive behavior. ${ }^{(12,27,28)}$

Depression in JME can be explained on the basis of 
the bio-psycho-social model: biological (endocrine related effects of seizure; metabolic effects of seizure; adverse effects of antiepileptic drugs); psychological (personality factors, individual's perception and attitude towards epilepsy and its treatment); social (stigma attached to epilepsy. psychosocial support burden of treatment, employment related issues, compromised quality of life due to epilepsy). ${ }^{(29)}$

The cause of anxiety disorders in JME can be summarized as of neurobiological origin, psychosocial origin, iatrogenic origin (drug induced), or a combination of the above factors. ${ }^{(30,31)}$ The results obtained in the study of O'Muircheartaigh et al. provide convincing evidence for abnormalities in a specific thalamo-cortical circuit, with reduced structural and task-induced functional connectivity, which may underlie the functional abnormalities in JME. ${ }^{(1)}$ Dysfunction in these areas results in deficits of concept formation, abstract reasoning, planning and self-regulation of behavior, and control of impulsivity and emotions. ${ }^{(31)} \mathrm{T}$. Chachua et al. ${ }^{(32)}$ found a highly significant dominance trait (aggression) in $\mathrm{Brd2}+/$ - haploinsufficient mice compared with the wild type, more pronounced in females. Brd2+/- mice of either sex did not differ from wild-type mice in spatial learning and memory tests. Compared with wild-type littermates, it was found that there were decreased numbers of GABA neurons in the basolateral amygdala, which is consistent with the increase in aggressive behavior. $\mathrm{Brd} 2+/$ - haploinsufficient mice showed no cognitive impairment but had behavioral traits similar to those found in JME patients (recklessness, aggression. ${ }^{(9,32)}$ It should be noted that Mendelian JME genes and nonMendelian risk alleles have not been detected in over $90 \%$ of affected patients. ${ }^{(33,34)}$

In several patients with JME, AEDs contribute to the psychiatric manifestations; however, it does not mean that antiepileptic drugs need to be stopped. The focus should be on proper selection of AEDs after assessing the risks and benefits. ${ }^{(35)}$

Comorbid anxiety and depressive symptoms in patients with epilepsy adversely affects the quality of life. ${ }^{(36)}$ Timely identification and appropriate intervention improves the quality of life of patients with nonpsychotic psychiatric disorder in epilepsy. ${ }^{(37)}$

Personality and nonpsychotic psychiatric disorder may be mild, moderate or severe, and patients with JME may have periods of "remission" where they function well. Mild disorders that do not seriously interfere with a person's ability to function socially are common. Severe disorders are rare in JME. However, whenever there is persistently impaired social functioning in conditions in which it would normally not be expected, the evidence suggests that this is more likely to be created by personality abnormality than by other clinical variables. ${ }^{(38)}$ It is very important to understand this, because these disorders can significantly disrupt the lives of both the affected patient with JME and those who care about that person. The disorders may cause problems with relationships, work or school, and can lead to social isolation, alcohol or drug abuse, or seizure aggravation.

The management and treatment of personality and nonpsychotic psychiatric disorders can be a challenging and controversial area, for by definition the difficulties have been enduring and affect multiple areas of functioning in JME patients. ${ }^{(39,40)}$

This often involves interpersonal issues, and there can be difficulties in seeking and obtaining help from organizations in the first place, as well as with establishing and maintaining a specific therapeutic relationship. Unfortunately, there are substantial social stigma and discrimination related to the diagnosis of personality and nonpsychotic psychiatric disorder in JME patients. There is always a need to maintain appropriate professional-personal boundaries, while allowing for emotional expression and therapeutic relationships.

A comprehensive psychiatric evaluation should be offered at the time of diagnosis to detect these comorbidities and to treat them. ${ }^{(22)}$ Different types of psychological therapies have been shown to help patients with JME and comorbidity PDs. There is no single approach that suits everyone; treatment should be tailored to the individual. Along with the professional treatment plan, we should discuss lifestyle and self-care strategies of patients with JME. Coping skills needed to adapt effectively to epilepsy are often lacking in many patients; this too needs to be improved in order to improve the quality of life. ${ }^{(41)}$

\section{Competing interests}

The authors declare that they have no competing interests.

\section{Conclusion}

Comorbid personality and nonpsychotic psychiatric disorders are a common and interdisciplinary problem in JME management. The disorders in patients with JME often go undiagnosed and hence untreated. Therefore, this problem requires further and extensive investigation. The neurologistepileptologist should be able to identify the comorbid disorders and understand that liaising with mental health professionals is beneficial.

\section{References}

1. O'Muircheartaigh J, Vollmar C, Barker GJ, Kumari V, Symms MR, Thompson P, et al. Abnormal thalamocortical structural and functional connectivity in juvenile myoclonic epilepsy. Brain. 2012;135 (Pt 12):3635-44. doi: 10.1093/ brain/aws296.

2. Wandschneider B, Thompson PJ, Vollmar C, Koepp MJ. Frontal lobe function and structure in juvenile myoclonic epilepsy: a comprehensive review of neuropsychological and imaging data. Epilepsia. 2012;53(12):2091-8. doi: 10.1111/ epi.12003.

3. Janz D. Juvenile myoclonic epilepsy. Epilepsy with impulsive petit mal. Cleve Clin J Med. 1989;56 Suppl Pt 1:S23-33; discussion S40-2

4. Janz D, Christian W. Impulsive petit-mal. Deutsche Zeitschrift f'ur Nervenheilkunde 176:346-386. (English translation by Genton P. 1994). In: Malafosse A, Genton P, Hirsch E, Marescaux C, Broglin D, Bernasconi M. (eds.) 1994;1957:229-251. 
5. Walsh J, Thomas RH, Church C, Rees MI, Marson AG, Baker GA. Executive functions and psychiatric symptoms in drug-refractory juvenile myoclonic epilepsy. Epilepsy Behav. 2014;35:72-7. doi: 10.1016/j.yebeh.2014.03.026.

6. Moschetta S, Fiore LA, Fuentes D, Gois J, Valente KD. Personality traits in patients with juvenile myoclonic epilepsy. Epilepsy Behav. 2011;21(4):473-7. doi: 10.1016/j. yebeh.2011.03.036

7. Lee JM, Kim SY, Hwang YH, Lee HW, Suh CK, Park SP. Longitudinal Assessment of Cognitive Function in Patients with Juvenile Myoclonic Epilepsy. J Korean Epilepsy Soc. 2008; 12(2):85-91.

8. Proposal for revised classification of epilepsies and epileptic syndromes. Commission on Classification and Terminology of the International League Against Epilepsy. Epilepsia. 1989;30(4):389-99.

9. Shilkina O, Artyukhov I, Moskaleva P, Strotskaya I, Shnayder N. Cognitive disorders in juvenile myoclonic epilepsy. International Journal of Biomedicine. 2017;7(1):914. doi: 10.21103/Article7(1)_RA1

10. de Araújo Filho GM, Lin K, Lin J, Peruchi MM, Caboclo LO, Guaranha MS, et al. Are personality traits of juvenile myoclonic epilepsy related to frontal lobe dysfunctions? A proton MRS study. Epilepsia. 2009;50(5):1201-9. doi: 10.1111/j.1528-1167.2009.02021.x.

11. Moschetta S, Valente KD. Impulsivity and seizure frequency, but not cognitive deficits, impact social adjustment in patients with juvenile myoclonic epilepsy. Epilepsia. 2013;54(5):866-70. doi: 10.1111/epi.12116.

12. de Araujo Filho GM, Yacubian EM. Juvenile myoclonic epilepsy: psychiatric comorbidity and impact on outcome. Epilepsy Behav. 2013;28 Suppl 1:S74-80. doi: 10.1016/j. yebeh.2013.03.026.

13. Martínez-Domínguez S, Labrada-Abella J, Pedrós-Roselló A, López-Gomáriz E, Tenías-Burillo JM. [Mental illness, personality traits and quality of life in epilepsy: control study of patients with juvenile myoclonic epilepsy and other epilepsies]. Rev Neurol. 2013;56(12):608-14. [Article in Spanish].

14. Somayajula S, Vooturi S, Jayalakshmi S. Psychiatric disorders among 165 patients with juvenile myoclonic epilepsy in India and association with clinical and sociodemographic variables. Epilepsy Behav. 2015;53:37-42. doi:10.1016/j. yebeh.2015.09.024.

15. Conrad R, Schilling G, Bausch C, Nadstawek J, Wartenberg HC, Wegener I, et al. Temperament and character personality profiles and personality disorders in chronic pain patients. Pain. 2007;133(1-3):197-209.

16. Pierò A. Personality correlates of impulsivity in subjects with generalized anxiety disorders. Compr Psychiatry. 2010;51(5):538-45. doi:10.1016/j.comppsych.2010.02.003.

17. Rzezak P, Moschetta SP, Lima E, Castro CX, Vincentiis $\mathrm{S}$, Coan AC, et al. Distinct domains of impulsivity are impaired in juvenile myoclonic epilepsy but not in temporal lobe epilepsy. Epilepsy Behav. 2015;45:44-8. doi: 10.1016/j. yebeh.2015.02.028.

18. Cloninger CR. A practical way to diagnosis personality disorder: a proposal. J Pers Disord. 2000;14(2):99-108.

19. Congdon E, Canli T. A neurogenetic approach to impulsivity. J Pers. 2008;76(6):1447-84. doi: 10.1111/j.14676494.2008.00528.x. Review.

20. Wandschneider B, Centeno M, Vollmar C, Stretton J, O'Muircheartaigh J, Thompson PJ, et al. Risk-taking behavior in juvenile myoclonic epilepsy. Epilepsia. 2013;54(12):2158-
65. doi: 10.1111/epi.12413.

21. de Araujo Filho GM, de Araujo TB, Sato JR, Silva Id, Lin $\mathrm{K}$, Júnior $\mathrm{HC}$, et al. Personality traits in juvenile myoclonic epilepsy: evidence of cortical abnormalities from a surface morphometry study. Epilepsy Behav. 2013;27(2):385-92. doi: 10.1016/j.yebeh.2013.02.004.

22. Coppola A, Caccavale C, Santulli L, Balestrini S, Cagnetti C, Licchetta L, et al. Psychiatric comorbidities in patients from seven families with autosomal dominant cortical tremor, myoclonus, and epilepsy. Epilepsy Behav. 2016;56:38-43. doi: 10.1016/j.yebeh.2015.12.038.

23. Ertem DH, Dirican AC, Aydın A, Baybas S, Sözmen V, Ozturk M, et al. Exploring psychiatric comorbidities and their effects on quality of life in patients with temporal lobe epilepsy and juvenile myoclonic epilepsy. Psychiatry Clin Neurosci. 2017;71(4):280-288. doi: 10.1111/pcn.12499.

24. Mosby's Medical Dictionary, 9th edition. Elsevier; 2009. 25. Thomas RH, Walsh J, Church C, Sills GJ, Marson AG, Baker GA, et al. A comprehensive neuropsychological description of cognition in drug-refractory juvenile myoclonic epilepsy. Epilepsy Behav. 2014;36:124-9. doi:10.1016/j. yebeh.2014.04.027.

26. Lin JJ, Mula M, Hermann BP. Uncovering the neurobehavioural comorbidities of epilepsy over the lifespan. Lancet. 2012 Sep 29;380(9848):1180-92. doi: 10.1016/S01406736(12)61455-X.

27. Camfield CS, Camfield PR. Juvenile myoclonic epilepsy 25 years after seizure onset: a population-based study. Neurology. 2009;73(13):1041-5. doi: 10.1212/WNL.0b013e3181b9c86f. 28. Caplan R, Siddarth P, Gurbani S, Hanson R, Sankar R, Shields WD. Depression and anxiety disorders in pediatric epilepsy. Epilepsia. 2005;46(5):720-30.

29. Miller JM, KustraRP, VuongA, HammerAE, Messenheimer JA. Depressive symptoms in epilepsy: prevalence, impact, aetiology, biological correlates and effect of treatment with antiepileptic drugs. Drugs. 2008;68(11):1493-509.

30. Beyenburg S, Schmidt D. [Patients with epilepsy and anxiety disorders. Diagnosis and treatment]. Nervenarzt. 2005;76(9):1077-8, 1081-2, 1084-6 passim. doi: 10.1007/ s00115-004-1867-4. [Article in German].

31. Kar SK, Tekkalaki B, Mohapatra S, Saha R. Mental Health Perspectives of Epilepsy: Focus on Anxiety Disorders. Delhi Psychiatry Journal 2015; 8(1):7-15.

32. Chachua T, Goletiani C, Maglakelidze G, Sidyelyeva G, Daniel M, Morris E, et al. Sex-specific behavioral traits in the Brd2 mouse model of juvenile myoclonic epilepsy. Genes Brain Behav. 2014;13(7):702-12. doi: 10.1111/gbb.12160.

33. Harden C. Juvenile myoclonic epilepsy-what does the future look like? Epilepsy Curr. 2014;14(3):150-1. doi: 10.5698/1535-7597-14.3.150.

34. Shnayder NA, Shilkina OS, Petrov KV, Chernykh IA, Diuzhakova AV. [Clinical and genetic heterogenity of juvenile myoclonic epilepsy]. Epilepsy and Paroxyzmal Conditions. 2016;8(2):20-36. doi:10.17749/2077-8333.2016.8.2.020-036. [Article in Russian]

35. Bell GS, Mula M, Sander JW. Suicidality in people taking antiepileptic drugs: What is the evidence? CNS Drugs 2009;23(4):281-92.

36. Kotov AS. [Anxiety in epileptic patients]. Zh Nevrol Psikhiatr Im SS Korsakova 2013;113(4 Pt 2):41-4.[Article in Russian]

37. Johnson EK, Jones JE, Seidenberg M, Hermann BP. The relative impact of anxiety, depression, and clinical seizure 
features on health-related quality of life in epilepsy. Epilepsia 2004;45(5):544-50.

38. Nur U, Tyrer P, Merson S, Johnson T. Relationship between clinical symptoms, personality disturbance, and social function: a statistical enquiry. Irish Journal of Psychological Medicine. 2004;21:19-22.

39. Pellock JM. Defining the problem: psychiatric and behavioral comorbidity in children and adolescents with epilepsy. Epilepsy Behav. 2004;5 Suppl 3:S3-9.
40. Pascalicchio TF, de Araujo Filho GM, da Silva Noffs MH, Lin K, Caboclo LO, Vidal-Dourado M, et al. Neuropsychological profile of patients with juvenile myoclonic epilepsy: a controlled study of 50 patients. Epilepsy Behav. 2007;10(2):263-7.

41. Asadi-Pooya AA, Schilling CA, Glosser D, Tracy JI, Sperling MR. Health locus of control in patients with epilepsy and its relationship to anxiety, depression, and seizure control. Epilepsy Behav. 2007;11(3):347-50. 